\title{
Retos en la formación para la docencia-investigación
}

\begin{abstract}
Resumen
El binomio docencia-investigación está sustentado por marcos normativos y postulados teóricos, que articulan o separan dichas funciones. El objetivo principal de este análisis busca reflexionar sobre la necesidad de articular la docencia con la investigación no solo en su función formativa, sino también en su función sustantiva. Hay estudios que han demostrado que la investigación mejora la calidad docente y la formación de los futuros profesionales, por lo que se debe potenciar la formación para la investigación y mejora continua en las competencias para ambas funciones. Por otra parte, la investigación científica puede conducir a El Salvador a mejorar la producción e innovación del conocimiento, en el que las instituciones de educación superior requieren no solo docentes investigadores con las competencias necesarias, sino también del apoyo del Estado y de la cooperación internacional. Para ello hay que revisar el marco normativo si este está potenciando esta actividad y la organización de la investigación en las universidades, respecto a las funciones de los docentes, para que ya no se vea separada de la investigación.
\end{abstract}

\section{Palabras clave}

Docencia, investigación, competencias, calidad de la educación.

\begin{abstract}
The teaching-research binomial is supported by policy frameworks and theoretical postulates, which articulates coordinate or separate these functions. The main objective of this analysis seeks to reflect on the need to link teaching with research, not only in its formative function, but also in their substantive role. Studies have shown that research improves teacher quality and training of future professionals, so training for research should be enhanced and continuous improvement in skills for both functions. Furthermore scientific research can lead El Salvador to improve the production of knowledge and innovation, where higher education institutions, require not only teacher-researchers with the necessary skills, but also the support of the State and international cooperation. This requires reviewing the regulatory framework, if it is promoting this activity and the organization of research in universities, regarding the roles of teachers, for them to no longer see separate teaching and research.
\end{abstract}

\section{Keywords}

Teaching, research, expertise, quality of education.

Blanca Ruth Orantes. Licenciada en Relaciones Internacionales por la Universidad de El Salvador, Máster en Docencia Universitaria de la Universidad Tecnológica de El Salvador, Certificado-Diploma de Estudios Avanzados y doctoranda en el Programa de Doctorado "Investigación e Innovación Educativa en la Educación Superior" bajo convenio entre la Universidad de El Salvador y Universidad de Granada, España. Su trabajo está orientado a la investigación e internacionalización de la educación superior. Actualmente se desempeña como directora de Investigaciones en la Utec. 


\section{Introducción}

En los últimos años se ha observado un interés creciente por la investigación científica, observando esfuerzos, identificando sistemas nacionales de más avanzada, en donde se ha establecido un perfil del docente que articula las diferentes actividades propias de la docencia y la investigación, con el objetivo de que incida en el desarrollo de la investigación científica y la calidad académica, así como en el estatus de las universidades y de los grupos de investigación e investigadores (Alonso, 1984).

También se encuentran otros sistemas nacionales en los que la práctica de la investigación científica como una profesión no es muy antigua y sus investigadores poseen solo nivel de grado y pocos con maestrías. En estos se experimenta un raquítico desarrollo de la ciencia -subdesarrollo científico-, que se nota en todas las áreas de conocimiento y que, a su vez, agudiza aún más la dependencia de otros países. Algunos de los programas de postgrado no generan investigación y no capacitan a los investigadores. Al respecto, Alonso (1984), en su estudio "Formación de investigadores en México", encuentra que el número de especialistas en investigación educativa (refiriéndose a esta área de conocimiento) es reducido. De ahí se subraya la escasez de preparación científica para la gran mayoría del personal de una universidad. Tampoco existe preparación específica del personal que trabaja en tareas de apoyo de la investigación educativa. Lo antes expuesto denota escasa formación para la investigación que requiere no solo contar con un doctorado, sino también dedicarse a la actividad investigadora. Para el caso de El Salvador, solo se cuenta con 15 doctores ( 12 hombres y 3 mujeres) a nivel de postgrado, que forman parte del personal investigador de las instituciones de educación superior (IES) que dedican como mínimo el $80 \%$ de su tiempo a esta actividad. Lo anterior podría ser uno de los factores causales de la baja productividad científica, pues El Salvador tiene el menor número de publicaciones, ocupando el último lugar en Centroamérica (Conacyt, 2012).

Otra posición respecto al tema es la planteada por De Hoyos (1981). Basa su análisis en que la formación se desarrolla en la práctica, sin menospreciar la preparación inicial formal, que es necesaria aun cuando la mayoría del profesorado universitario no se incorpora a las actividades de investigación por no estar preparado para las actividades de investigación y docencia. Esta caracterización explica que existan deficiencias en la formación de investigadores; su calificación académica investigadora, en algunas universidades, refleja que aún no hay una política de formación de investigadores y personal de apoyo. Ante tal situación, se debe promover la formación académica del personal de investigación, el rigor metodológico, la mejor definición de resultados y la calidad académica de los proyectos (Alonso, 1984), pues "la investigación puede convertirse en un excelente recurso formativo en la formación inicial y permanente del profesorado" (Forner, 2000 , p. 33), y, por supuesto, incide en la calidad académica, utilizando la investigación como herramienta de aprendizaje o aprendizaje por proyectos, mejora la calidad académica y prepara a los estudiantes para el mundo laboral (Gómez, 2014).

Las posturas anteriores (Alonso, 1984; De Hoyos, 1981; Forner, 2000) son en principio la premisa de análisis de competencias docentes y de investigación, que se presentan en este artículo; si debe ser una práctica de todo docente o son funciones diferentes. Uno de los retos que se presentan en el marco normativo de El Salvador inicia en el enunciado del Art. 53 de la Constitución de la República de El Salvador (1973): "El Estado propiciará la investigación y el quehacer científico". La Ley General de Educación (2004), dentro de los objetivos generales de la educación nacional, plantea, en el Art. 3 literales b) y c), que los planes de estudio deben equilibrar planes y programas sobre la base de la ciencia y además cultivar la imaginación creadora y el desarrollo de la capacidad crítica, que no es otra cosa más que el deber de articular la docencia con la investigación, que en el nivel superior, en la misma ley, el Art. 27 expresa que se deberá "promover la investigación en todas sus formas" (p.15).

Así, la mencionada Ley de Educación Superior asume el mandato como uno de sus objetivos en el Art. 2, literal b). Además, como función sustantiva, establece en el Art. 3.: "La educación superior integra tres funciones: La docencia, la investigación científica y la proyección social". El literal segundo establece que "la docencia busca enseñar a aprender, orientar la adquisición de conocimientos, cultivar valores y desarrollar en los estudiantes habilidades para investigación e interpretación, para su formación integral como profesionales" (р.36). Este mandato normativo, que establece la disposición anterior, debe interpretarse como un deber de articular la docencia con la investigación, es decir, la investigación como función formativa es un eje transversal en el proceso enseñanza-aprendizaje, que 
se convierte en una herramienta para la formación de competencias profesionales., Y en el caso de la proyección social —que no es objeto de este artículo, pero que resulta importante mencionar-, es la función para sensibilizar sobre su entorno al futuro profesional. En algunas instituciones de educación superior se la denomina investigación de cátedra o investigación en el aula.

La investigación, como función sustantiva, está contemplada en forma expresa en el mismo Art. 3 de dicha ley, en el literal tercero: "La investigación es la búsqueda sistemática y análisis de nuevos conocimientos para enriquecer la realidad científica, social y ambiental, así como para enfrentar los efectos adversos del cambio climático" (p.36). Esta función establece, de acuerdo con el artículo 37, ordinal d), que las instituciones de educación superior deberán:

Realizar o mantener, por 10 menos, un proyecto de investigación relevante por año, en las áreas que se ofrecen; para lo cual deberán contar con presupuesto asignado y podrán ser apoyados con recursos públicos y privados. Los proyectos de investigación con duración mayor de un año deberán reportar al Ministerio de Educación el avance anual de los mismos (p.47).

En este mismo orden, el ordinal g) expresa que "los proyectos de investigación (...) serán asumidos, preferentemente, por los docentes a tiempo completo" (p.48). Sin embargo, en la realidad, la investigación es realizada por investigadores a tiempo completo y docentes investigadores, dedicados a tiempo parcial a la investigación. Este punto denota cómo la docencia es vista como una función diferente de la investigación, en los sistemas de educación superior de otros países; y en El Salvador no es la excepción. Esta situación obedece a postulados teóricos que a continuación se presentan y que tienen que ver con las competencias y papeles de los profesores universitarios.

\section{Competencias docentes y/o de investigación}

Aunque la investigación en los últimos años ha ocupado las agendas en debates académicos, reconociendo su importancia, en la realidad se observa aún en los sistemas universitarios separada la labor docente de la investigación, como función principal la primera, por lo que los docentes no se sienten comprometidos ni motivados a realizar actividades de investigación, que van desde dirigir investigación en el aula, tesis de grado y postgrado, proyectos de I+D, formar parte de grupos de investigación, entre otras. Al respecto se plantean estudios que sustentan las competencias docentes y de investigación. Por ejemplo, en España, la propuesta realizada por Zabalza (2003) atribuye al profesorado universitario competencias profesionales como: planificar el proceso enseñanza-aprendizaje, seleccionar y preparar los contenidos disciplinarios, ofrecer información comprensible y organizada, manejar las nuevas tecnologías de la información, diseñar la metodología y organizar las actividades que se deben realizar, mantener comunicación y relacionarse con los alumnos y tutorizar, evaluar, reflexionar e investigar sobre la enseñanza, identificarse con la institución y trabajar en equipo.

El desempeño del docente universitario exige que lo acompañen determinadas actitudes hacia las poblaciones con las que trabaja. Entre ellas, puntual, disciplinado, acucioso, solidario, servicio de calidad, equidad, ético, identidad con la familia, profesión, etc. Se suma que ha de ser un profesional con sólidos conocimientos de la ciencia y tecnología, así como de cultural general, que no son otra cosa, que las competencias a formarse en su papel como docente universitario. Además, garantizar el desempeño profesional del docente, así como las habilidades y destrezas reales y potenciales que tiene que desarrollar con rigor científico; un alto compromiso de cambio para asegurar los servicios con equidad y calidad. Los profesores universitarios expertos deben mostrar: dominio del conocimiento, eficacia en la resolución de problemas y la intuición de problemas que permitan fortalecer el proceso educativo utilizando la investigación como herramienta de aprendizaje (González, 2002).

Entonces, si las competencias docentes son factor clave para alcanzar la calidad educativa, se requiere de un programa formativo que facilite el proceso de socialización de los profesores principiantes o noveles, y que permita revisar y fortalecer las prácticas docentes de profesores de mucha experiencia, como recurso de mejora y aseguramiento de la calidad educativa, identificando las necesidades en formación y actualización pedagógica y en habilidades sociales (Sánchez y Mayor, 2006). Para estas autoras se hace necesario en este programa de formación, contar con profesores mentores que se convierten en modelos ya experimentados, que cuentan con competencias personales y competencias profesionales. Es decir, preparar talento para el cambio generacional, con amplias competencias personales y profesionales: 
Profesores comprometidos, flexibles y tolerantes, entusiastas, transparentes, empáticos, reflexivos, seguros y dinámicos. Habilidosos en la reflexión y en el análisis de la enseñanza, demuestran tener éxito en sus clases, disponen de habilidad para enseñar y trabajar con colegas, saber cómo interactuar con diversas personalidades, conocen los trucos de la profesión, comprenden la cultura profesional, son valorados por colegas y alumnos y dominan los conocimientos propios de la disciplina (p. 930).

En la escala de competencias sintetizadas por Sánchez y Mayor (2006) resulta natural que el profesor novel al inicio de la función docente universitaria exista inseguridad en las actividades que realiza, por ello se caracterizan por un nivel de complejidad y crítico en los primeros años de ejercicio docente, así lo plantean en su estudio Herrera, Fernández, Caballero y Trujillo (2011), además señalan que:

En el contexto universitario actual y el nuevo Espacio Europeo de Educación Superior (EEES) parece emerger un reclamo conjunto que insta a la necesaria planificación de propuestas para acoger, formar y facilitar las tareas propias del ejercicio docente en profesores noveles (p. 215).

El reclamo antes mencionado coincide con lo planteado por Escudero (1999): al profesor universitario se le exige y reconoce: dominio de conocimientos de sus respectivas áreas de conocimiento y competencias demostradas en proyectos y publicaciones. Se le concibe como creador y constructor del saber. Por su parte, Sánchez (2008) establece que a los profesores universitarios se les debe formar con un nivel básico, otro especializado y, además, de investigación. Relacionado con lo anterior, hay autores que plantean la importancia de estas competencias y los contextos de enseñanza-aprendizaje en que los profesores universitarios actúan; tienen implicaciones relevantes, puesto que existe un componente para propiciar mayor rendimiento académico (De la Fuente, Martínez, Peralta, y García, 2010).

Así, De La Fuente y Justicia (2007), en un estudio enfocado en el modelo Didepro, aportan una visión comparada de la regulación como variable psicológica desde el proceso de enseñanza-aprendizaje, lo cual lo genera el contexto, pero principalmente el profesor como factor clave para que el estudiante se adapte, pues son las competencias e ideas previas del profesor las determinantes. Este modelo plantea la regulación multinivel e interactiva del proceso enseñanza-aprendizaje sobre las variables: presagio (inician con el enfoque preferido), proceso (diseño, desarrollo y evaluación), producto (influencia de las variables presagio y proceso), es decir, antes-durante-después. La influencia en los modos de estudio: enfoque profundo, enfoque superficial y enfoque de logro. Este modelo plantea una solución ante la falta de regulación de la enseñanza y del aprendizaje, pues existe una influencia de la Teoría de los Enfoques de Aprendizaje (SAL), que hace explícita la influencia de la autorregulación del aprendizaje e incluye la regulación de la enseñanza.

El modelo Didepro resulta altamente interesante, pues plantea, entre otros, la variable producto, que permite evaluar para diseñar estrategias de aprendizaje que incidan directamente en la calidad del aprendizaje (Herrera, Jiménez y Castro, 2011). Así, también en un estudio realizado por De la Fuente, Pichardo, Justicia, y García-Berbén (2008), se concluyó que "un aprendizaje más profundo va acompañado de mayor planificación, debido a que los datos verificaron la existencia de una relación negativa entre el enfoque superficial y la autorregulación del aprendizaje" (p. 33).

Básicamente, Perrenoud (2001) propone algunos criterios que deben responder a una formación profesional de alto nivel: el primero plantea la transposición didáctica fundada en el análisis de las prácticas y de sus transformaciones. Un referencial de competencias que permita identificar los saberes y capacidades requeridas en cada nivel. Elaborar un plan de formación organizado en torno a competencias. Aprendizaje a través de problemas en un procedimiento clínico y articulación efectiva entre la teoría y práctica, entre otros.

Considerando estos criterios, "la formación de profesores debe orientarse hacia un aprendizaje a través de problemas, enfrentar a los estudiantes a la experiencia de clase y trabajar a partir de observaciones, de su asombro, de sus éxitos y fracasos" (Perrenoud, 2001, p. 12). Por ello, este autor considera que el docente no solo debe tener competencias académicas, sino también aspectos psicológicos, porque hay momentos que el profesor debe imponer la calma a los grupos en clase, enfrentar grupos heterogéneos, estudiantes equilibrados y en otras ocasiones con doble vida, en conflictos laborales, familiares y hasta de conflictos de personalidad. 
Otra creencia que se considera un mito es que los buenos investigadores son buenos docentes (Terenzini y Pascarella, 1994). Lo correcto sería que para ser un buen docente se necesitase investigar; pero creer que la calidad de la enseñanza de un profesor fluye directamente de su trabajo como investigador no puede ser tan acertado porque ambas funciones son actividades diferentes y exigen conocimientos y habilidades distintos (Gibbs, 1995), lo cual no significa que no estén relacionados. Sí plantea que el profesor universitario debe tener pleno dominio de su disciplina y poseer requisitos para enseñarla.

Para De Miguel (2003), no hay estándares de calidad que definan la función docente de un profesor universitario. El criterio común es la competencia que demuestra en el desarrollo de su trabajo en el aula; considerando que el profesor desempeña distintas tareas y papeles, como la docencia, la investigación y la gestión académica. Todo lo expuesto implica que cada vez es mayor la exigencia de un desempeño eficaz e innovador. Tal como plantea Tedesco (1996), la formación inicial recibida y las exigencias actuales dejan al descubierto muchas debilidades, principalmente porque hay una diferencia entre la formación pedagógica y la formación científica, y no solo diferencia sino también una separación en el desempeño docente.

Un estudio realizado por Gilis et al. (2008) establece que se requiere un perfil de competencias con el enfoque centrado en el estudiante, para responder a los nuevos enfoques del constructivismo en la educación superior, en el que seobserva un cambio pedagógico ya no centrado en el profesor, sino en el estudiante. El estudio valida un perfil de competencias basado en conocimientos, habilidades y actitudes, los cuales deben estar presentes desde la formación inicial del profesor. Lo cierto es que la formación pedagógica implica el dominio de la materia y de estrategias pedagógicas, no únicamente el dominio de transmitir la información, lo que se observa con frecuencia (Tedesco, 1996). En cambio, la formación científica permite transmitir no solo los procesos cognitivos que exige cada disciplina, sino que el docente que, además de dominar la pedagogía, conoce el método científico y lo aplica, puede convertirse en un guía y modelo del proceso de aprendizaje, el cual adquiere su máxima importancia y donde es posible articular la formación pedagógica con la científica. El ejercicio de la docencia, por tanto, requiere de procesos de reconversión permanente. Para ello, se deben poseer competencias cognitivas y personales que tan solo un proceso relativamente largo de formación inicial puede otorgar. Esta actualización permanente es a la que respondió la Declaración de Lovaina (Cemes, 2009).

En un estudio realizado por Herrera, Fernández, Caballero y Trujillo (2011) distinguieron tres tipos de competencias docentes en acción educativa: "Competencias docentes destinadas a la preparación de la docencia, competencias docentes durante la docencia y competencias docentes posteriores a la docencia" (pp. 224-225). Las competencias docentes destinadas a la preparación de la docencia se refieren a toda la fase de planificación que requiere esta función; las competencias durante la docencia a las habilidades y destrezas que se requieren en la ejecución de lo planeado y la función sustantiva de la docencia en que se facilita o transfiere el conocimiento; y las competencias docentes posteriores, que es también importante no solo por la reflexión de la función, sino por el reto y necesidad que requiere la práctica docente de actualizar e innovar el conocimiento, lo que implica investigación educativa y propuestas de mejora continua. Por ello, dada la importancia del desarrollo de las competencias del docente tiene en el desempeño de su trabajo, que es una tarea completa que implica conocimiento intenso, habilidades, acciones y sobre todo compromisos para la innovación (Madero y Valenzuela, 2012).

Para algunos autores, como Ojeda y López (2005), un país se desarrollo requiere que se presenten varios factores, entre ellos que los individuos cuenten con una formación de alta calidad. Esta formación requiere de las competencias en investigación, cuyo desarrollo se posibilita dentro de los programas de formación de doctorado, puesto que estos estudios "pueden entenderse como una formación de investigadores en cualquier rama de la ciencia" (Morón, 2005, p. 22). El doctorado de postgrado es el último y más alto grado académico que conceden las universidades, el cual viene a ser como el referente del conocimiento pleno y acabado en una materia (Sevilla, 2005). En esta dirección, tal y como apunta Buela-Casal (2005), Ios programas doctorales y el número de tesis doctorales producidas por una universidad es un indicador significativo del grado de calidad académico-científico alcanzado. No significa lo anterior que sea el único criterio válido para considerar a una universidad de calidad. Lo que sucede es que el número de universidades de cada país que figuran en el ranking mundial está directamente relacionado con los porcentajes de producción científica. 
Según Buela-Casal, Bretón y Agudero (2005), no se reflexiona sobre las consecuencias importantes que tiene un programa de doctorado en el desarrollo de los distintos sectores de un país o región, su impacto en la universidad, en el desarrollo industrial, capacitación profesional y mejoras en el sistema educativo, en general. Para estos autores, en los países del primer mundo la investigación y el desarrollo industrial son inseparables; si no se investiga no hay avance industrial, que es lo que puede generar la riqueza de un Estado. Por ello, cada vez más en países desarrollados, las empresas, públicas y privadas dedican fondos para la investigación. Desde esta perspectiva, nace la necesidad de formar y especializar investigadores. Con ello se busca incidir directamente en los cambios sociales, económicos y políticos de un país. "El doctorado es el mayor grado académico que se puede conseguir en las universidades, lo que debe equivaler a un conocimiento acabado y pleno en una materia" (Buela-Casal y Castro, 2008, p. 49).

En El Salvador existen muchas universidades que no cuentan con programas propios de doctorado, otras que tienen doctorados transnacionales, y escasas con doctorados propios. Sobre esta base se presenta, a continuación, un análisis de diversos enfoques de la formación de investigadores.

La formación de los investigadores debe ser capaz de transformar y educar. La formación inicial como docente investigador debe desarrollarse con las siguientes características: ser activo y crítico, tener un comportamiento reflexivo y racional, emitir juicios y toma decisiones, tener capacidad para modelar actitudes y aptitudes en los estudiantes.

De lo anterior se plantea que los docentes deben poseer competencias y habilidades intelectuales y sociales (Forner, 2000). Esas habilidades permiten un papel del docente que se desplace de la simple transmisión del conocimiento a su creación e innovación. Al respecto, Sautu (1997, p. 180) resalta a "la investigación como una forma de conocimiento que se caracteriza por la construcción de evidencia empírica -apoyada en un cuerpo teórico- a partir de la aplicación de reglas de procedimiento rigurosas y explícitas", lo cual se puede atribuir a las competencias de un investigador.

Sautu (2003) señala que, en todo proceso de investigación social, teoría, objetivos y metodología deben articularse entre sí. Plantea que la interacción docente-estudiante constituye el eje para la construcción del conocimiento. El intercambio de experiencias forma parte constitutiva de nuestra propia práctica de investigación y docencia. Para Pirela y Pritero (2006) la educación debe ir más allá de la transmisión de conocimientos, debe ser capaz de generarlos, a través de la conformación de ideas, la práctica de la innovación y su aplicación para la propensión de cambios por medio de la intervención de la realidad social. Para ello, debe contribuir a formar individuos que sean capaces de aprender por sí mismos, que sepan investigar, cuestionar críticamente, innovar, abiertos a los cambios y con autonomía intelectual, sensibles a los problemas sociales, comprometidos con la comunidad, país y región, y conscientes de sus responsabilidades sociales. De esta forma, la investigación es una tarea fundamental de todo profesional, ya que a través de ella se estudian los factores que intervienen en las realidades sociales, con el fin de intervenir en ellas. Los docentes universitarios, como investigadores, deben desarrollar las competencias con énfasis en el dominio de los términos, procesos y teorías de campo de la investigación, fundamentadas en el razonamiento científico, que le permita abordar de manera crítica la realidad, construir mapas cognitivos y valorativos que la expliquen, utilizar la capacidad de análisis y síntesis, juicio crítico, motivación al logro, entre otros, para la generación de nuevos conocimientos.

Por otra parte, el estudio de Justice et al. (2006) establece que la investigación es una potente herramienta pedagógica en la educación superior, pues permite que los estudiantes se conviertan en participantes en cursos basados en la investigación. Por ello, el profesor debe dominar las competencias en investigación, contando así con la oportunidad de aplicar las habilidades basadas en aquellas. Aunque este estudio analiza que en las ciencias sociales aumenta el aprendizaje por indagación; y, en contrario, en disciplinas de gerontología, antropología y geografía disminuye, lo que obedece al tipo de investigación de acuerdo con el área de conocimiento. El mismo estudio plantea, como un punto de tensión, si la investigación se debe aprender en un curso independiente 0 un enfoque pedagógico, o en todo caso en ambas, pues en las actividades investigativas se requiere de las competencias genéricas y específicas.

Hasta aquí se han expuesto posturas y competencias señaladas por diferentes autores, pero que relacionados con el contexto de un programa de formación de 
investigadores de muchas universidades en Latinoamérica, y particularmente en El Salvador, no son la excepción. Las universidades, en este contexto, hay pocas que cuentan con doctores, lo que podría constituir una de las causas por las que los resultados en investigación son escasos y con un bajo índice de calidad e impacto, además de que el proceso de investigación está lleno de plagio y falseamiento de datos, entre otros. Esta situación inquieta tanto a las autoridades y directivos como al propio profesorado. La mejora es lenta, aun cuando se observa la necesidad, cada vez mayor, de contar con verdaderos programas de formación de investigadores que permitan mejorar la calidad universitaria y otros fenómenos propios de la eficiencia e integración de las funciones docentes e investigadoras.

Los estudios de doctorado pueden entenderse, en este sentido, como una formación de investigadores dirigida a licenciados universitarios en cualquier rama de la ciencia y que, una vez finalizada, podrá permitir al individuo especializarse en un área determinada de conocimiento y convertirse en un experto en ese campo (Morón, 2005). Se reconoce que el doctorado es el último y más alto grado académico que conceden las universidades, algo que viene a ser como el referendo del conocimiento pleno y acabado en una materia (Sevilla, 2005), por lo que se debe pensar en crear programas de doctorado, ya sea de forma conjunta con otras universidades nacionales, de forma transnacional o por iniciativa propia de cada universidad para formar a los investigadores del más alto nivel, los cuales posibilitarán el desarrollo de la ciencia local. Si las diferentes capacitaciones, cursos, diplomados y maestrías abonan a la formación de investigadores, el doctorado es el nivel deseable e idóneo para formar verdaderos investigadores.

Otro aspecto es señalado por García-Pérez y Ayres (2012), quienes identificaron como problemas las altas tasas de deserción y capacitación a los estudiantes sobre el proceso de investigación que debían realizar en el doctorado con investigadores y supervisores experimentados. En estos talleres se discutieron y desarrollaron en conjunto un modelo del proceso de investigación, con la finalidad de ayudar a que los estudiantes desarrollaran una aproximación más completa y reflexiva respecto a sus trabajos. El estudio identificó que los estudiantes, a pesar de tener un buen entendimiento de su área de estudio, generalmente tienen poca exposición al proceso de descubrir algo nuevo. También demostraron tener poca apreciación de que gran parte del proceso de investigación involucra persistencia, hacerle frente a contratiempos, buscar metódicamente otras alternativas, etc., resultando beneficiados de poder discutir y comparar sus experiencias en un ambiente relativamente no estructurado, lo que lleva a los autores a proponer que facilitar este tipo de discusiones (estudiantesinvestigadores) ayuda a expandir sus experiencias de investigación y, como los estudiantes manifestaron, ayuda a aclarar ideas. Este estudio demuestra el porqué la experiencia de los doctorados se considera el proceso ideal para la formación en investigación.

Considerando que "el desarrollo de la humanidad depende de la evolución cultural científica y técnica, y esto se consigue en los centros de conocimiento e investigación que son las universidades, encargadas de expandir el conocimiento a las nuevas generaciones de estudiantes" (Bermúdez et al., 2011, pp. 6-7), se puede afirmar que las universidades de El Salvador deben aprovechar el recurso humano existente. En este marco, la reunión de Bolonia estableció la independencia y autonomía a las universidades, "necesarias para la investigación, que debe estar orientada siempre a las necesidades de los individuos y a los cambios de la sociedad" (Declaración de Bolonia, 1999). Y, más recientemente, la Declaración de BudapestViena (2010), que marca el final de la primera década del Proceso de Bolonia y que supuso la presentación oficial del Espacio Europeo de Educación Superior (EEES), tal como lo plantean Herrera, Fernández, Caballero y Trujillo (2011), "ha modificado considerablemente el mapa y la geografía de la educación superior en Europa, al tiempo que ha planteado, entre otras, cuestiones relativas a todos aquellos temas relacionados con la calidad de la docencia, la formación del profesorado y su desarrollo profesional" (p. 216). Las instituciones de educación superior en Europa han tenido el compromiso de modificar las ordenaciones académicas, las metodologías docentes, la conexión con el mercado de trabajo, la investigación, la transferencia del conocimiento y la responsabilidad social, dando para con ello a la estrategia Universidad 2015 del gobierno de España (Fernández y Vigil, 2010):

Lo último respecto a este tema es que la Comisión Europea emitió un comunicado de prensa sobre resultados de la reunión en Bruselas, el 25 de abril de 2012, en donde la Comisaría Europea de Educación, Cultura, Multilingüismo y Juventud, Androulla Vassilou, ha dicho a los estados miembros que deben modernizar urgentemente sus sistemas de educación superior 
y eliminar los obstáculos para lograr un EEES que funcione a pleno rendimiento en respuesta a la crisis $y$ al aumento del desempleo juvenil, se debe actuar para ayudar a impulsar el empleo y el crecimiento en Europa.

El objetivo sigue siendo que la EEES funcione a pleno rendimiento y proporcione una enseñanza de primera clase y capacidades de empleo para todos, estimule la innovación y garantice un reconocimiento adecuado de las cualificaciones académicas. Siendo la educación superior elemento central de los esfuerzos para superar la crisis, se deberá garantizar una financiación suficiente para que la educación superior haga una contribución duradera al bienestar económico y al progreso social. La comunicación a los ministros del proceso de Bolonia considera que la educación superior es clave para el empleo y el crecimiento. El grupo de seguimiento de Bolonia cuenta con una agenda de modernización en relación con la educación superior; señala cinco ámbitos clave de reforma:

Aumentar el número de titulados a fin de que, a más tardar en 2020, se cumple el objetivo, establecido en Europa 2020, de que haya un $40 \%$ de jóvenes con cualificaciones de educación superior; aumentar la calidad y hacer que la educación superior sea más acorde a las necesidades de los puestos de trabajo y a las demandas sociales; mejorar la calidad de la movilidad para el estudio; integrar la educación superior en el triángulo del conocimiento de la educación, la investigación y el desarrollo; y mejorar la gobernanza y la financiación. El proceso de Bolonia y la agenda de modernización de la Comisión se refuerzan mutuamente (Comunicado de prensa Bruselas, 2012).

La experiencia en el EEES resulta valiosa, evidenciando que el triángulo del conocimiento contempla la investigación y, lógicamente, la formación de doctores para mejorar esta función sustantiva. No obstante, los cambios en el EEES siempre han tenido incidencia en Latinoamérica. En El Salvador, no se han considerado los cambios, aún existen muchos licenciados, ingenieros y arquitectos que egresan sin haber cursado ninguna materia sobre metodología de la investigación y, en menor medida, haber realizado al menos un proyecto de investigación. Este hecho resulta paradójico cuando un rasgo definitivo de las universidades es la necesaria relación entre la educación superior y la investigación. Lo ideal sería que todo titulado se formara en las herramientas básicas de la investigación a lo largo de su carrera (Bermúdez et al., 2009). Sin embargo, en muchos sistemas educativos no se contempla, al menos en Latinoamérica.

También se encuentra la oportunidad de pertenecer a redes para buscar la oportunidad de intercambio y movilidad, que podría convertirse en una oportunidad para el aprendizaje y la formación. Como afirma Sevilla (2005):

La movilidad fomenta la calidad académica y permite que la diversidad se convierta en una ventaja, además de mejorar la capacidad para el empleo. Los gobiernos deben salvar los obstáculos actuales a la movilidad, otorgando incentivos a los estudiantes, asesoramiento académico y personal, el aprendizaje de idiomas y el reconocimiento de títulos. Se debe mejorar las salidas profesionales para jóvenes investigadores y profesores, favoreciendo también sus salidas al exterior. Así como favorecer el intercambio al exterior (p. 20).

En El Salvador son pocas las universidades que han iniciado los procesos de internacionalización. No obstante, se debe trabajar en una cultura en este sentido, lo que implica fortalecer capacidades docentes para estar acorde a un perfil internacional de intercambio, o en todo caso aprovechar estos procesos de ventajas de las instituciones de educación superior mejor posicionadas en los rankings por los perfiles de sus docentes.

\section{Reflexiones}

En las instituciones de educación superior se hace investigación. No obstante, esta actividad es poco visible en medios de difusión internacionales que permitan aparecer a El Salvador como un país que promueve la ciencia, pues en algunos rankings no aparece y en otros ocupa los últimos lugares. En cuanto a la apuesta en formación para la investigación, el bajo número de doctores denota que no se ha aprovechado las oportunidades de becas para doctorados que ofrecen la cooperación internacional, y lograr que estos procesos se articulen con la actividad investigadora en las IES, por lo que se debe reflexionar en apostarle al del desarrollo de la ciencia y tecnología, pues, aunque se aprobó La Ley de Desarrollo en ciencia y Tecnología (2013), aún no se observa efecto potenciador. 
En cuanto a la Ley de Educación Superior (2004), el Art. 38, establece que es el personal académico y que esta disposición legal los menciona como "encargados de la investigación", sin señalar cuál debería ser el perfil para realizar dicha actividad, por lo que se observa que no está regulado, y se deja a criterio de las instituciones de educación superior. En este sentido, el personal académico responde a la clasificación hecha por Álvarez-Rojo et al. (2009), quien plantea que existen perfiles académicos que responden a las categorías de especialistas en docencia, docente investigador, profesor asociado y docente multifunción.

En cuanto al modelo de enfoque de las instituciones, responden a un modelo profesionalizante o de formación profesional bajo el enfoque por competencias que, según Álvarez-Rojo et al. (2009), es un centro de formación profesional al servicio del mercado de trabajo, por lo que no se tiene énfasis en la investigación, aunque ya se explicó que la Ley de Educación Superior (2004) establece a la investigación como una función sustantiva y que aparece como una de las funciones de toda universidad. No obstante, la calidad académica no se visualiza como un componente que puede potencializarse a través de la actividad investigativa como refuerzo a una enseñanza de gran calidad. Aunque existen esfuerzos por organizar la investigación, se identifica como debilidad la forma de asignar los créditos por investigación, por lo que no se observa como un incentivo.

Por otra parte, aún no se asumen las nuevas tendencias dictadas en el proceso de Bolonia, en donde se destacó "el papel fundamental de la comunidad académica. (Declaración de Budapest-Viena, 2010), escenario en que se encuentran, entre otros, profesorado e investigadores; y continúa el reto en Bruselas (2012), en donde la investigación es un factor clave.

El Viceministerio de Ciencia y Tecnología ha promovido una agenda nacional que integra problemas y necesidades. Lo anterior es fruto de un esfuerzo conjunto, cuya finalidad es que las instituciones de educación superior y centros de investigación pongan el énfasis en el desarrollo de investigación científica, considerando las condiciones de infraestructura y de recursos humanos, así como propiciar la investigación para el desarrollo de las diferentes áreas estratégicas vinculadas con el plan de gobierno 2009-2013, que por cierto es tiempo de revisar dicha agenda para el próximo quinquenio. Además, se aprobó la Ley de Desarrollo en Ciencia y Tecnología, que busca fortalecer la investigación científica. Así también promover el apoyo con un fondo para investigación, que cada año apoye las propuestas de investigación científica, lo que requiere entonces reactivar el Fondo de Investigación para la Educación superior y dignificar la labor de investigación como parte de las actividades docentes.

Los retos son, aumentar los programas de formación para la investigación científica, apoyar a todas las instituciones de educación superior con programas de financiamiento anual que permitan desarrollar proyectos pertinentes en la investigación básica, la investigación aplicada y la investigación para el desarrollo, procurando la innovación y vinculación con el sector industrial, como factor de desarrollo nacional. Además, aumentar la publicación científica por medios arbitrados, que visibilicen la producción e innovación del conocimiento, cuyos destinatarios son la comunidad académica y científica, entre otros, además de abonar al perfil de la institución y del profesor (docenteinvestigador), que son carta de presentación a la hora de solicitar cooperación para proyectos de investigación y/o cooperación.

Algunas instituciones de educación superior ya han iniciado acciones de fortalecimiento institucional. Las demás deberán aumentar sus esfuerzos para contribuir a la ciencia; un reto para los académicos y una deuda hacia el país. Los resultados de la producción científica se evidencian en la siguiente tabla, en la que aparecen algunas de las universidades de El Salvador que, relacionadas con 23 universidades, 7 institutos especializados y 6 institutos tecnológicos (Conacyt, 2012), su posición es muy baja. 
Tabla 1

Producción científica de El Salvador años 2010 al 2013

\begin{tabular}{lccc}
\multicolumn{1}{c}{ Institución que aparecen en el ranking SIR } & $\mathbf{2 0 1 0}$ & $\mathbf{2 0 1 1}$ & $\mathbf{2 0 1 2}$ \\
\hline Universidad de El Salvador (UES) & 23 & 36 & 34 \\
Universidad Centroamericana José Simeón Cañas (UCA) & 4 & 10 & 14 \\
Universidad Tecnológica de El Salvador (UTEC) & 2 & 5 & 4 \\
Universidad Dr. José Matías Delgado (UJMD) & 4 & 3 & 10 \\
Universidad Evangélica de El Salvador (UEES) & 0 & 4 & 2 \\
Universidad Salvadoreña Alberto Masferrer (USAM) & 0 & 3 & 3 \\
Universidad Don Bosco (UDB) & 0 & 1 & 3 \\
Escuela Superior de Economía y Negocios (ESEN) & 0 & 1 & 4 \\
\hline
\end{tabular}

Fuente: SIR, Ranking Iberoamericano 2010, 2011 y 2012. SCI mago Research Group. Recuperado de www.scimahoir.com

Los datos presentados, independiente de otros rankings, miden la producción científica, la cual es posible cuando ha sido validada por árbitros de la comunidad científica, que mide, entre otros, rigurosidad en el método, uso de normas técnicas, originalidad, coherencia, etc. Además, el ranking SIR determina los índices de calidad e impacto. Entonces, el reto ya no es solo formar docentes investigadores, sino, también, que ellos comuniquen los resultados de sus investigaciones en medios que permitan medir la calidad de la investigación y el aporte a la ciencia.

\section{Referencias bibliográficas}

Alonso, J.A. (1984). Formación de investigadores de la educación en México. Revista de la Educación Superior, 13(52). Recuperado de http://www.anuies. mx/servicios/p_anuies/publicaciones/revsup/res052/ txt12htm.

Álvarez-Rojo, V., Asensio-Muñoz I., Clares, J., del-Frago, R., García-Lupión, B., García-Nieto, N., García-García, M., Gil, J., González-González, D., Guardia, S., Ibarra, M., LópezFuentes, R., Rodríguez-Diéguez, A., Rodríguez-Gómez, G., Rodríguez-Santero, J., Romero, S., y Salmerón, P. (2009). Perfiles docentes para el espacio europeo de educación superior (EEES) en el ámbito universitario español. RELIEVE, 15(1), 270-283. Recuperado de http://www.uv.es/RELIEVE/v15n1/RELIEVEV15n1_1. htm. Recuperado el 12/02/2012

Bermúdez, M.P., Castro, A. Sierra, J.C., y Buela-Casal, G. (2009). Análisis transnacional de los estudios de doctorado en el EEES. Revista de Psicodidáctica, 14, 193-210.

Bermúdez, M.P., Guillén-Riquilme, A., Gómez-García, A., Quevedo-Blasco, R., Sierra, J.C., y Buela-Casal, G. (2011).
Análisis del rendimiento en el doctorado en función del sexo. Educación XXI, 14(1), 17-33.

Buela-Casal, G. (2005). Situación actual de la productividad científica de las universidades españolas. International Journal of Clinical and Health Psychology, 5(1), 175-190.

Buela-Casal G., Bretón L.J., y Agudero D. (2005). Manual práctico para hacer un doctorado. Madrid: Editorial EOS.

Buela-Casal, G., y Castro, A. (2008). Análisis de la evolución de los programas de doctorado con mención de calidad en las universidades españolas y pautas para su mejora. Revista de Investigación en Educación, 5, 49-60. Recuperado de http://webs.uvigo.es/reined/

Comunicado de Prensa. Bruselas (2012).

Conacyt, Consejo Nacional de Ciencia y Tecnología (2012). Indicadores de Ciencia y Tecnología 2011. San Salvador, El Salvador: Departamento de Desarrollo Científico y Tecnológico.

Constitución de la República de El Salvador (1983). Diario Oficial Número 234, Tomo 281 del 16/12/1983. Reformas hasta Junio 2009. D.L. No. 36 27/5/2009. D.O. 102, Tomo 383 4/6/2009.

Declaración de Bolonia (1999). Bolonia, 19 de junio de 1999. Recuperado de www.educacion.es

Declaración de Budapest-Viena (2010). Recuperado de http://www.eees.es/es/documentacion

Cemes (2009) Declaración de Lovaina. Recuperado de http://www.icbf.gov.co/portal/page/portal/PortallCBF/ Especiales/SRPA/DLovaina-2009.pdf

De Hoyos, F. (Coord.) (1981). Los estudios sobre la investigación educativa en México (1979-1980). En Congreso Nacional de Investigación Educativa, Vol. 1. (pp. 543-594). México. 
De la Fuente, J., Martínez, J.M., Peralta, F.J., y García, A.B. (2010). Percepción del proceso enseñanza-aprendizaje y rendimiento académico en diferentes contextos instruccionales de la educación superior. Psicothema, 22(4), 806-812.

De Miguel, M. (2003). Calidad de la enseñanza universitaria y desarrollo profesional del profesorado. Revista de Educación, 331, 13-34.

De la Fuente, J., y Justicia, F. (2007). El modelo DIDEPROÒ de regulación de la enseñanza y del aprendizaje: avances recientes. Electronic Journal of Research in Educational Psychology, 5, 535-564. Recuperado de http://www.investigacion-psicopedagogica.org/revista/ articulos/13/espannol/Art_13_209.pdf.

De la Fuente, J., Pichardo, M.C., Justicia, F., y García-Berbén, A.B. (2008). Enfoques de aprendizaje, autorregulación $\mathrm{y}$ rendimiento en tres universidades europeas. Psicothema, 20(4), 705-711.

Escudero, J.M. (1999). La formación permanente del profesorado universitario, cultura, política y procesos. Revista Universitaria de formación del profesorado, 34, 133-157.

Fernández, A.M., y Vigil, M.A. (2010). Bolonia 46. Bolonia 2010. La construcción del Espacio Europeo de Educación Superior. En Vicedecanato de Extensión Universitaria, Estudiantes, Cooperación al Desarrollo y Relaciones Institucionales de la Facultad de Educación y Humanidades de Melilla (Ed.), V Jornadas de Experiencias en Educación: La proyección social del docente del siglo XXI (pp. 519-526). Melilla: GEEPP Ediciones.

Forner, Á. (2000). Investigación educativa y formación del profesorado. Revista Interuniversitaria de Formación del Profesorado, 39, 33-50.

García-Pérez, A., y Ayres, R. (2012). Modelling research: a collaborative approach to helping PhD students develop higher-level research skills. European Journal of Engineering Education, 37(3), 297-306.

Gibbs, G. (1995). The relationship between quality in research and quality in teaching. Quality Higher Education, 1(2), 147-157.

Gilis, A., Clement, M., Laga, L., y Pauwels, P. (2008). Establishing a competence profile for the role of student-centred teachers in Higher Education in Belgium. Research in Higher Education, 49(6), 531-554.

Gómez C., V.M. (2014). Calidad, docencia y evaluación en educación superior. Universidad Nacional de Colombia en Congreso anual Docente de la Universidad Tecnológica de El Salvador, enero 2014.
González, V. (2002). ¿Qué significa ser un profesional competente? Reflexiones desde una perspectiva psicológica. Revista Cubana de Educación Superior, XXII(1), 45-53.

Herrera, L., Fernández, A.M., Caballero, K., y Trujillo, J.M. (2011). Competencias docentes del profesorado novel participante en un proyecto de mentorización. PROFESORADO. Revista de Currículum y Formación del Profesorado, 15(3). Recuperado de http://www.ugr. es/ recfpro/rev153COL2.pdf.

Herrera, L., Jiménez, G., y Castro, A. (2011). Aprendizaje del alumnado universitario de primer y último curso de las titulaciones de Psicología y Magisterio. Electronic Journal of Research in Educational Psychology, 9(2), 659-692. Recuperado de http://www.investigacionpsicopedagogica.org/revista/articulos/24/english/ Art_24_555.pdf.

Justice, C., Rice, J., Warry, W., Inglis, S., Millar, S., y Sammon, S. (2006). Inquiry in Higher Education: Reflections and Directions on Course Design and Teaching Methods. Innovative Higher Education, 31(4), 201-214.

Ley de Desarrollo en Ciencia y Tecnología de la República de El Salvador (2013). Decreto No. 381, Asamblea Legislativa. D.O. No.96, Tomo No. 399. 28 de mayo del año 2013.

Ley de Educación Superior de la República de El Salvador (2004). Reformas 2011. Diario Oficial, Tomo No. 391. San Salvador, El Salvador (9 de junio 2011).

Ley General de Educación Superior (1996) Reformas junio 2005. D.O. No. 108, Tomo No. 367, del 13 de junio de 2005.

Madero C.A., y Valenzuela, B. (2012). Evaluación de competencias docentes para la mejora de la calidad de la educación superior en México. UnilaSalle Editora, 1(20). Recuperado de http:revistas.unilasalle.edu.br/ index,php/Dalogo/article/view/277

Moron, I. (2005). Competencias de los docentes universitarios en sus funciones académicas. Tesis Doctoral en Ciencias de la Educación, Universidad Rafael Belloso Chacín.

Ojeda, C., y López, P.L. (2005). Sobre formación de investigadores. En Séptimo Congreso Internacional Retos y Expectativas. México: Universidad de Guanajuato. Recuperado de http://congreso\%201/mesa\%20G/mesa_g_5.pdf

Perrenoud, P. (2001). La formación de los docentes en el siglo XXI. Revista de Tecnología Educativa, XIV(3), 503523 Recuperado de http://www.unige.ch/fapse/SSE/ teachers/perrenoud/php_main/php_2001/2001_36. $\underline{\text { html }}$ 
Pirela De Faria, L., y Pritero de Alizo, L. (2006). Perfil de competencias del docente en la función de investigador y su relación con la producción intelectual. Opción, 22(50), 110-121.

Sánchez, A. (2008). Elementos clave en el diseño de módulos y titulaciones EEES. Revista de Investigación en Educación, 5, 41-48. Recuperado de http://webs. uvigo.es/reined/

Sánchez, m., Y mayor, c. (2006). Los jóvenes profesores universitarios y su formación pedagógica. Claves y controversias. Revista de educación, 339, 923-946.

Sautu, R. (1997) Acerca de qué es y no es investigación científica en Ciencias Sociales. México: Fondo de Cultura Económica.

Sautu, R. (2003). Todo es teoría: objetivos y métodos de investigación. Buenos Aires: Lumiere.

Sevilla, D. (2005). ¿Qué es un doctorado? Pautas para realizarlo. En G. Buela-Casal (Ed.), Manual práctico para hacer un doctorado. Madrid: EOS Universitaria.
SIR, Ranking Iberoamericano (2010). SCI mago Research Group. Recuperado de www.scimaholab.com

SIR, Ranking Iberoamericano (2011). SCI mago Research Group. Recuperado de www.scimaholab.com

SIR, Ranking Iberoamericano (2012). SCI mago Research Group. Recuperado de www.scimagoir.com

Tedesco, J.C. (1996). Fortalecimiento del rol de los docentes: balance de las discusiones de la $45^{a}$ Sesión de la Conferencia Internacional de Educación. Ginebra, Suiza: Oficina Internacional de la Unesco. Recuperado de www.apagina.pt

Terenzini, P., y Pascarella, E. (1994). Living with myths. Change, 1, 28-32.

Zabalza, M. (2003). Competencias docentes del profesorado universitario. Calidad y desarrollo profesional. Madrid: Narcea.

Zabalza, M.A. (2007). Competencias docentes del profesorado universitario. Calidad y desarrollo profesional $\left(2^{\mathrm{a}}\right.$ Edición). Madrid: Narcea. 